\title{
Gianni Celati e la fotografia come «pratico pensare per immagini»
}

In dialogo con Ghirri e Gajani

\section{Marina Spunta}

\section{(2) OpenEdition \\ Journals}

Edizione digitale

URL: https://journals.openedition.org/cher/714

DOI: $10.4000 /$ cher.714

ISSN: 2803-5992

\section{Editore}

Presses universitaires de Strasbourg

Edizione cartacea

Data di pubblicazione: 9 juillet 2020

Paginazione: $97-112$

ISBN: 979-10-344-0068-3

ISSN: 1968-035X

\section{Notizia bibliografica digitale}

Marina Spunta, «Gianni Celati e la fotografia come «pratico pensare per immagini»», reCHERches

[Online], 24 | 2020, online dal 20 septembre 2021, consultato il 17 novembre 2021. URL: http:// journals.openedition.org/cher/714 ; DOl: https://doi.org/10.4000/cher.714 


\title{
Gianni Celati e la fotografia come "pratico pensare per immagini» In dialogo con Ghirri e Gajani
}

\author{
MARINA SPUNTA ${ }^{1}$
}

$\mathrm{F}$ in dagli anni Sessanta Gianni Celati si avvicina alla fotografia, un'arte di cui, a posteriori, ammette "non sapevo quasi niente» (2004a: 86), e stabilisce uno stretto dialogo con vari fotografi, in particolare con Carlo Gajani, artista e fotografo bolognese, poi con Luigi Ghirri, la cui opera diventa un punto di riferimento importante nei suoi testi narrativi e cinematografici, e in vari saggi critici, editi ed inediti. Celati conosce Ghirri nei primi anni Ottanta, in seguito al suo invito di scrivere delle «descrizioni di paesaggi che entrassero in risonanza con la loro ricerca» (85), cioè con la ricerca dei fotografi coinvolti da Ghirri in Viaggio in Italia, un progetto del 1984 che ha ridefinito la fotografia italiana contemporanea. Ne risultano il palinsesto generativo che è Verso la foce, pubblicato prima in due testi brevi del 1984 e 1987 e poi in volume nel 1989, e i nuclei di novelle e racconti pubblicati negli anni Ottanta e successivamente - Narratori delle pianure, Quattro novelle sulle apparenze e Cinema naturale. Avvalendomi del ricco materiale d'archivio del Fondo Celati della Biblioteca Panizzi a Reggio Emilia e del Fondo Gajani a Bologna ${ }^{2}$, in questo saggio considererò la riflessione celatiana sulla fotografia, e sulla risonanza fra fotografia e scrittura e descrizione del paesaggio, nel contesto di un più ampio discorso sulle immagini e sulla visione. Prendendo spunto da quella che Michele Vangi definisce la «modalità riflessiva» del rapporto tra letteratura e fotografia, atta a "constatare che di solito, la riflessione letteraria sulla fotografia sollecita un'autoriflessione, cioè una riflessione [...] sulle possibilità e limiti della letteratura stessa» (2005: 39), valuterò la funzione generativa che la fotografia ricopre nell'opera celatiana, quale mezzo per aprirsi in modo nuovo alla visione

1 Marina Spunta, University of Leicester.

2 Mi riferirò ai due fondi con le abbreviazioni FC e FG, ed alla numerazione delle pagine usata nei vari manoscritti. Ringrazio Carmelo De Luca della Biblioteca Panizzi per la professionalità e disponibilità. 
del paesaggio negli anni Ottanta, e, non meno importante, per riflettere sulla (propria) scrittura e rimodellare la propria immagine autoriale in quegli anni. Dopo un breve spoglio della biblioteca celatiana sulla fotografia, considererò la consonanza di alcuni scritti celatiani per e su Ghirri e Gajani - i due fotografi con cui ha avuto una più stretta collaborazione - e la loro risonanza con testi editi e manoscritti di Verso la foce. La mia analisi mostrerà come Celati intenda la fotografia come una guida per "pensare lo spazio esterno» (Celati 2017d: 24) e come "pratico pensare per immagini», in linea con l'estetica ghirriana, ovvero, nelle parole di Jerry Thompson, «[as] a new kind of epistemology, a new [...] way of learning about the world» (2016: 12), un approccio che pone l'artista/ scrittore nel ruolo di «an attentive observer, a willing participant in [...] a system larger than the artist's individual, personal, particular needs» (14).

Come per Ghirri e Gajani, l'interesse di Celati per la fotografia va iscritto all'interno di un più ampio interesse verso le arti visive e di un discorso di continuità fra i codici, che ne garantisce tuttavia l'indipendenza. Se Ghirri pensava alla fotografia come arte dell'immagine in dialogo con varie arti e discipline e come "frutto di una serie di relazioni tra i diversi mondi della comunicazione» (Ghirri 2010: 7), dalla pittura, al cinema, all'immagine pubblicitaria - come rivela nelle sue Lezioni di fotografia, e come si evince dal suo lavoro, a partire dal suo primo libro, Kodachrome del 1978 - similmente Celati iscrive la fotografia, a fianco della scrittura, in un più ampio discorso sulle immagini, i meccanismi della visione, le arti visive, i rituali collettivi, la percezione e l'esperienza dell'esterno. L'idea celatiana di fotografia - come per Ghirri - si basa sulla tradizione delle arti visive, del cinema (soprattutto Antonioni, Fellini, Rossellini, Wenders), e su esempi classici quali la collaborazione tra Strand e Zavattini per Un paese, sulla fotografia tedesca, inglese e soprattutto americana, e su alcuni testi teorici chiave, da Roland Barthes a John Berger, con cui Celati ha collaborato a lungo. Ciò emerge da uno spoglio della biblioteca celatiana conservata presso la Biblioteca Panizzi, che in circa duecento volumi offre un interessante spaccato di alcune delle letture fatte dall'autore negli anni Ottanta e Novanta. Oltre a volumi sull'arte (ad esempio su Piero della Francesca, Cézanne, Brueghel, Friedrich) o testi teorici di varie discipline, colpisce il numero limitato di testi sulla fotografia: una ventina di volumi, tra libri fotografici, teorici e cataloghi, pubblicati dagli anni Settanta ai primi anni Novanta, oltre ad una decina di volumi di e su Ghirri. Troviamo ad esempio libri su fotografi americani ed europei, da Ansel Adams a Paolo Monti, come pure sulla fotografia locale e sul Po. I testi teorici includono fra gli altri l'edizione inglese di Camera chiara di Roland Barthes ed alcuni volumi pubblicati da Linea di Confine per la Fotografia Contemporanea, organizzazione emiliana che dal 1989 organizza progetti internazionali di analisi del territorio ${ }^{3}$. I cataloghi comprendono per lo più mostre di fotografia

3 Su Linea di Confine si vedano i saggi di Roberta Valtorta e William Guerrieri in Valtorta 2013. Altri testi celatiani sulla fotografia includono Federico Arborio Mella, Sulla strada della fotografia, testo poco noto ma citato come essenziale in Marra 2001, 
contemporanea a cui Ghirri aveva partecipato, tra cui Napoli '81: sette fotografi per una nuova immagine, ma anche Nuovo paesaggio Americano, importante mostra sui Nuovi Topografi organizzata da Paolo Costantini nel 1987 a Venezia, oltre a progetti di fotolibri che hanno coinvolto sia Ghirri che Celati, quali Traversate del deserto e Il racconto del nostro presente.

Nella biblioteca celatiana della Panizzi sono inoltre presenti molti testi di scrittori con cui Celati mostra una consonanza di vedute in quegli anni, in primo luogo Italo Calvino e Peter Handke: i testi più sottolineati ed annotati dall'autore sono Palomar, Se una notte d'inverno un viaggiatore e Lezioni Americane di Calvino, e Lento ritorno a casa di Handke, soprattutto in passaggi che riguardano la visibilità e la descrizione del paesaggio. Se per Calvino la parola scritta nasce "prima come ricerca d'un equivalente dell'immagine visiva», come suggerisce in «Visibilità» (1988: 89), unico passaggio sottolineato da Celati in questa lezione americana, per Celati, come si evince in un brano inedito "Sulle immagini», la "possibilità di apparizione delle immagini resta sempre una possibilità immaginaria» (FC 11/114), un tentativo, o, per citare un termine ricorrente nei titoli dei libri di Handke, un Versuch, che in tedesco indica sia tentativo che saggio critico. Per Celati, come per Ghirri, la capacità di vedere queste «apparizioni» e di riprodurle in scrittura, come in fotografia, non è un dono dato a tutti solo perché si hanno gli occhi, ma offerto solo a chi trovi una Stimmung con il luogo, una consonanza con i paesaggi descritti, una risonanza (emotiva) che sottintende un dialogo fra pari e quindi un ascolto. Di conseguenza, la descrizione di un luogo che Celati auspica sia nella fotografia che nella scrittura deve accordarsi alle immagini evocate dal luogo stesso, come avviene nel lavoro di Ghirri. Nel saggio "Commenti su un teatro naturale delle immagini», posto a introduzione al fotolibro ghirriano Il profilo delle nuvole, Celati dichiara che il testo «non è un documentario fotografico sulla situazione storica d'un paesaggio italiano, ma piuttosto sui modi di guardare già previsti in un paesaggio, e sulle loro risonanze affettive» (4 settembre). Per Celati, come per gli scrittori sopracitati, l'apertura all'immagine, in particolare quella fotografica, rappresenta sia un'apertura al visivo e ad altri codici, che un un approccio fenomenologicamente nuovo all'esterno e alla descrizione. Nella lezione «Leggerezza», un testo pesantemente annotato nella biblioteca celatiana, Calvino sostiene: "Voglio dire che devo cambiare il mio approccio, devo guardare il mondo con un'altra ottica, un'altra logica, altri metodi di conoscenza e di verifica» (1988: 9) - termine che rimanda al titolo di un'importante serie fotografica di Ugo Mulas, Le verifiche (19711972), chiave di volta autoriflessiva per la fotografia italiana, proprio come le Lezioni americane lo sono per la letteratura, ed altre discipline. Celati sembra fare altrettanto nell'aprirsi alla fotografia quale nuova epistemologia, ma anche, contrariamente a Calvino, e in parte proprio in risposta ad una letteratura che

Ian Jeffrey, Photography, ed il catalogo della mostra sui 150 anni della fotografia in Italia. Un itinerario. 
egli reputa troppo cerebrale ed asfittica, come quella di Calvino ${ }^{4}$, quale metodo di lavoro sul campo, come passaggio dall'idea alla pratica dell'arte, fatta sul momento, in linea con la pratica fotografica - come suggerisce Elisabetta Rasy in una delle prime recensioni a Verso la foce (1989: 25) - un approccio che permette a Celati di dar vita a una nuova modalità narrativa imperniata su una maggiore risonanza con il paesaggio.

In linea con scrittori e fotografi a lui affini, in vari saggi editi ed inediti, l'autore iscrive la fotografia in un più ampio discorso sul "pensare per immagini» che permetta di vedere e descrivere l'esterno con una lingua «necessaria», che esuli dal pensiero discorsivo ma si apra invece all'indeterminatezza. Nel saggio del 1992 «Soglia per Luigi Ghirri», Celati definisce la fotografia di Ghirri come «pratico pensare per immagini», "che è anche il pensiero del limite della misura», una "misura scalare che pone ogni cosa nella sua distanza, la distanza delle cose da noi» e che, in contrasto al «pensiero discorsivo [...] con le sue spiegazioni e valutazioni», "ci permette di far tesoro dell'indeterminatezza...» (Celati 2004b: 194). Con «pensare per immagini» Celati riprende una frase spesso usata da Ghirri per definire il «senso del [suo] lavoro», ad esempio nel saggio di introduzione a Kodachrome (Ghirri 1997: 21), una frase forse ispirata al titolo di un articolo di Gillo Dorfles trovato per strada e da Ghirri ripreso in una nota fotografia, Roma 1978. In quest'articolo il critico sostiene che il pensiero per immagini ci può far avvicinare alla realtà nascosta delle cose più del pensiero logico-scientifico - un approccio consono a quello di Ghirri e Celati. Per Celati, la «soglia del lavoro di Ghirri» è «ben rappresentata da quei due alberi piantati nella nebbia», in cui «[c]'è ordine anche se c'è nebbia» (Celati 2004b: 194), quindi da una dialettica apparentemente impossibile: da una lato l'inquadratura, che sospende «frammenti del visibile» (193) e ci permette appunto di vedere, dall'altro la nebbia, come «l'estrema ricerca d'una misura», quella dell'infinito (a cui Ghirri tendeva) e della «respirazione della terra» (194). Se per Ghirri questa ricerca, nelle parole di Giorgio Messori, tende «a vedere oltre il visibile, o meglio a vedere il pensiero che abita il visibile che si è voluto inquadrare» e a farci «entrare in uno spazio di immaginazione e memoria» (Messori 1992: 193), così per Celati questa «misura scalare» «ci permette [...] di affidarci ad apparizioni che non sappiamo bene cosa possano significare, ma che a volte diventano una misura per vedere tutto il resto» (Celati 2004b: 194). Similmente, nel saggio inedito su Wittgenstein «La media oscurità dell'esperienza», Celati definisce l'esperienza dell'esterno come «metro di misura, ma dipendente da qualcosa che è il contrario delle certezze, ossia l'esitazione» e come «un apprendimento a immaginare delle possibilità» (FC $1 / 2$ paragrafo 19), rivelando una chiara risonanza tra la sua riflessione sulla fotografia e quella sulla percezione e esperienza dell'esterno dello stesso periodo. Il "pensare per immagini» - che Celati a sua volta definisce «un modo ricettivo del pensiero» nella recensione

4 Si veda il giudizio di Celati su Calvino nel simposio in suo onore pubblicato nel 1987 (Celati 1987b). 
ai saggi di Ghirri, «Luigi Ghirri. Leggere e pensare per immagini» pubblicata da Marco Sironi (Celati 2004c: 205) - è alla base della riflessione di entrambi in quegli anni, come suggerisce anche Ennery Taramelli (2005: 27), ed in linea con il dibattito teorico del tempo sulla fotografia e sulle discipline dell'immagine e della visione, da Barthes a Eco al già citato Dorfles, come si evince ad esempio dall'antologia di scritti teorici sulla fotografia raccolta da Marra (2001). Allo stesso modo, il «pensare per immagini» permette a Celati di accostare vari codici e media, dalla fotografia al cinema alla scrittura, in vari saggi (quali «Commenti a un teatro naturale delle immagini» e «Collezione di spazi») e in numerosi testi autografi, tra cui gli appunti frammentari sull'immagine del faldone 2/35 (1992-93 ca.), dove il nome di Ghirri appare a fianco di vari artisti o scrittori (da Masaccio a Antonioni, da Ariosto a Leopardi, da Vermeer a Giacometti, per citarne solo alcuni) presi a modello per un'arte del narrare per immagini. Similmente, in un appunto del 10 giugno nel contenitore $12 / 145$, testo datato da Palmieri 1980-1984 circa, Celati riflette sul continuum e il discreto e sulla scrittura, il cinema e la fotografia e si domanda in uno schemino se siano mezzi di comunicazione, azione o descrizione. Questo schema è seguito da due equazioni: "Wenders = il cinema; Ghirri = la foto», ulteriore conferma della centralità dell'opera ghirriana nel pensiero celatiano sulla fotografia.

Fra le tante citazioni di Ghirri come sinonimo di fotografia e di un'arte che pone l'«esperienza» come modo di «sentire-pensare-immaginare» («La media oscurità dell'esperienza», FC 1/2) e le immagini come «necessarie», troviamo un appunto del 1981, nel faldone 12/167, scritto nel 1981 in Rue Simon-le-Franc:

Non frasi ma immagini, che siano come una materia anonima, le immagini nette delle fiabe ad esempio. Non frasi che sono già lì per commentare, mettere nomi e aggettivi sulle cose, ma immagini che vanno o portano verso un pezzo di storia naturale senza necessità di commenti. Queste immagini e descrizioni delle nostre storie naturali ci commuovono. Ė quella la materia anonima e collettiva. Occorre semplicità. (FC 12/167)

Questo brano rivela il desiderio di una lingua che passi rapidamente sulle cose, senza doverle nominare, quindi un narrare per immagini, in linea con quello che Handke nel saggio Il peso del mondo definisce «esperienza della liberazione da forme di scrittura già note» (1981: 7). Il frammento ricorda inoltre un brano che appare nella prima versione di Verso la foce, in cui Celati desidera di poter descrivere dettagliatamente le case alla foce del Po senza ingabbiarle nelle parole:

Vorrei fosse possibile che le parole passassero rapidamente attraverso tutte le diversità dell'esterno, così come fa lo sguardo, distinguendole una ad una. Che non ci fossero più "case sulla strada di Ca' Venier", ma quella laggiù, quell'altra, quel campo; e poterle descrivere tutte minutamente, per pagine e pagine (1984: 31).

Siamo vicini all'idea ghirriana di «immagine necessaria», citata nel dattiloscritto del 1987, «Pensando a un'immagine necessaria», in cui definisce la «fotografia come desiderio, immagine dialettica, e forse utopia» (Ghirri 1997: 118). Tale definizione fa eco all'idea celatiana di un narrare in cui «le spiegazioni sono quasi escluse» ma «c'era però [anche] il senso d'una connivenza 
nell'illusione delle parole, d'un legame che viene dall'immaginare le cose assieme, dove non ci sono più confini tra il verosimile e l'inverosimile» (Celati in Belpoliti e Cortellessa 2008: 35), come nei racconti orali di Giacomo Cangemi registrati negli anni Settanta (34). È importante sottolineare come per Celati il «pensare per immagini necessarie» venga accostato sia alla fotografia che al narrare orale e venga contrapposto alla scrittura e al "pensiero discorsivo", quale metodo rigido e lineare che non contempli indeterminatezza o digressioni analogiche spazio-temporali.

È utile ora accostare la riflessione celatiana sulla fotografia di Ghirri e sul "pratico pensare per immagini», a quella finora rimasta in ombra su Carlo Gajani, vista la collaborazione con entrambi i fotografi a progetti di descrizione del paesaggio padano negli stessi anni, molti dei quali rimasti incompiuti, ma non per questo meno interessanti. Nel far ciò non intendo glissare sulle profonde differenze dell'opera di Ghirri e di Gajani, né sul valore della collaborazione tra Celati e Gajani negli anni Settanta, oggetto di recenti studi (Palmieri 2017), ma appunto gettare luce su un tema ancora non trattato - il progettato lavoro sul paesaggio di Celati e Gajani. Mi propongo inoltre di riflettere sulla risonanza del pensiero celatiano su Ghirri e su Gajani, che leggo come conferma della costanza dell'idea celatiana sul "pensare-immaginare» e sulla funzione della fotografia nella sua narrativa. Celati frequenta Gajani fin dagli anni Sessanta, collabora con lui a vari progetti e inoltre vive per un periodo di tempo tra il 1978 e il 1979 a casa Gajani in via de' Castagnoli, a Bologna, come confermato da Angela Zanotti Gajani. Come ci ricorda Pasquale Fameli «la sintonia tra i due autori emiliani nei primi anni Settanta è fortissima» (2017: 447), visti i comuni interessi, in particolare sulla bagarre, il corpo, i deliri psicotici, gli «oggetti soffici», la ritualità quotidiana - temi che pur esulando dal presente studio, costituiscono elementi chiave dell'opera celatiana e della sua esperienza interartistica, in stretta continuità con la fase degli anni Ottanta. Il sodalizio tra Celati e Gajani apre a Celati nuove prospettive, quali quelle sulla pop art e l'informale, come ci ricorda Palmieri (2017: 432), ma viene interrotto senza portare a termine vari progetti. Tra questi troviamo «Disegni di paesaggio. Viaggio fotografico nelle pianure dell'ultimo Po», a cura di Renzo Renzi, con interventi di Gianni Celati, Antonio Faeti e Filiberto Menna, per una mostra a Rimini poi non organizzata (FG, dattiloscritto, 15 Giugno 1984), e un fotolibro incompiuto con Gajani, in quanto Celati abbandona il progetto per passare a lavorare con Ghirri. Il primo progetto doveva essere «Un libro fotografico sui paesaggi delle pianure che circondano l'ultima valle del Po, da Pontelagoscuro al mare», incentrato sull'arte di Gajani e teso a mettere in luce come «l'esperienza del paesaggio europeo e del paesaggio Padano possa essere una via dello sguardo, della visione e della ricerca artistica» (1), e come la fotografia debba essere intesa non "come reportage o illustrazione al servizio di un testo», ma come «un modo autonomo di raccontare il paesaggio» (1). L'«esplorazione [...] della valle padana come parte del continente europeo» (1) è qui contrapposta criticamente al «paesaggio americano divulgato da tutti i mass-media» (1-2) come «l'unico paesaggio possibile» (2). Quale secondo 
fine il volume intendeva investigare «il possibile uso della fotografia e indirizzo della ricerca artistica europea, rivolta al mondo esterno in cui viviamo, per definire l'immagine ambientale» (2); tale ricerca è vista in parallelo alla «ricerca in atto nel cinema, nella letteratura, nella fotografia» (2) - un approccio che riecheggia nel lavoro con Ghirri. Il volume avrebbe dovuto includere, oltre alle fotografie di Gajani, un'introduzione di Renzi, un intervento di Celati dal titolo «Il paesaggio europeo e il paesaggio americano nelle fotografie di Carlo Gajani» (2), un testo di Faeti su Dürer e la memoria, e uno di Menna su «Fotografia e pittura di paesaggio» (3). Pur nella sua forma embrionica, questo testo risulta molto indicativo in quanto conferma come, anche nella collaborazione tra Celati e Gajani, la fotografia faccia parte di un più ampio dibattito su arti visive, letteratura e paesaggio, che tuttavia sancisca la fotografia come arte autonoma, e un'arte che esprima una crescente attenzione al recupero del territorio locale, supportata in quegli anni da nuove politiche territoriali regionali e da progetti fotografici che ne beneficiano, tra cui molti voluti da Ghirri (Orlandi 2014).

Oltre a questo testo inedito, Celati scrive due brevi saggi su Gajani, «I viandanti osservatori», pubblicato nel 1983 sul pieghevole della mostra di Gajani Il viandante osservatore, e "Brani di natura nella valle del Po», apparso nel catalogo della mostra Carlo Gajani. Brani di natura nella valle del Po, tenutasi alla Galleria d'Arte Moderna a Ferrara nel 1985; entrambi i testi, conservati presso il Fondo Gajani sono stati recentemente pubblicati in Animazioni e incantamenti, da cui cito per maggior reperibilità. Celati inizia entrambi i saggi con un parallelo tra scrittura e fotografia, il che rivela come le sue riflessioni sulla fotografia siano funzionali a quelle sulla letteratura e tese a stabilire un nesso tra le due arti: «Dalla fotografia i narratori contemporanei hanno imparato varie cose. Hanno imparato prima di tutto a capire che la percezione è l'avventura che riguarda la nostra vita in ogni momento, e non c'è bisogno di introdurre nel mondo storie più monumentali che non riguardano nessuno» (Celati 2017b: 345). Così, secondo Celati, le fotografie di Gajani presentano i grattacieli di New York come «liberati dalla modernità» (345) e si aprono invece a «un'attesa (felice) di casi minimi» (348). Celati loda l'opera del «viandante osservatore» Gajani, per un ritorno alle «forme semplici» (334-347), in linea con il ritorno alla fiaba nella narrativa contemporanea, e per aver fatto "pulizia di tutti gli stereotipi» (347) della modernità, al fine di «restituirci ogni immagine come se fosse vista o raccontata per la prima volta» (347). Commenti simili risuonano nei saggi celatiani su Ghirri e rivelano la coerenza dell'idea di fotografia celatiana: ne Il mondo di Luigi Ghirri Celati celebra la fotografia ghirriana degli inizi per aver reso "forme semplici» e per l'allegria che ne scaturisce; Ghirri definisce a più riprese la propria opera come un tentativo di vedere il mondo per la prima volta con gli occhi di un bambino, e ricorre più volte all'immagine delle fiabe, spesso ripresa da Celati, ad esempio in "Commenti su un teatro naturale delle immagini» (15 settembre). La fotografia di Gajani e la fotografia in generale sembrano offrire a Celati un modello per superare la modernità, con 
i suoi dogmi, quali l'interiorità o l'inconscio, tramite l'uscire «fuodi di noi, nel paesaggio» (Celati 2017b: 345), verso l'«esteriorità del mondo» (348).

In «Brani di natura nella valle del Po», scritto dopo l'esperienza di Viaggio in Italia, Celati parte ancora da un parallelismo tra scrittura e fotografia per criticare l'eccessivo professionismo e l'autorialismo come «una forma di disastro mentale: [che] induce a dimenticare il mistero di queste attività, le quali diventano specializzazioni senza nessun contenuto, a parte i trucchi tecnici ed estetici» (2017c: 349), esternando la sua ammirazione per le fotografie di Gajani in quanto "non d'autore». Reduce dal lavoro per Viaggio in Italia non stupisce che Celati trovi un pregio nella (apparente) non autorialità dell'opera, data dal lavoro di collaborazione e dalla lontananza a un'idea di fotografia come Arte, già messa in luce da Arturo Carlo Quintavalle nel saggio introduttivo al volume Viaggio in Italia, e sottolineata successivamente da altri critici, fra cui Giorgio Messori (2004). Celati riserva un giudizio simile anche per l'opera di Ghirri, che celebra per la sua natura artigianale e non-autoriale, per la sua aderenza all'esperienza delle cose, quale arte a tutto campo, in dialogo con altre pratiche artistiche. Come quella di Ghirri, la fotografia di Gajani è portata ad esempio in quanto lontana da un'idea di un «bottino» di visioni da catturare, ma piuttosto come «intimità con le cose rappresentate, mettendo fuori gioco tutto ciò che è sensazionale e troppo rappresentativo» (Celati 2017c: 350), a partire da luoghi visti da tutti ed «anonimi ritagli di mondo» (350). È questa l'idea della qualsiasità che informa l'opera di Ghirri e di altri fotografi a lui contemporanei, tra cui il coetaneo Guido Guidi che la teorizza per il progetto Viaggio in Italia, quale attenzione alle cose più vicine a noi, alla nostra esperienza quotidiana, e con cui Celati trova una consonanza. Similmente, la «luce discreta», "dell'inverno o del tramonto» (350), rispettosa «delle ombre e delle forme» (350), che Celati rileva nelle fotografie di Gajani ci ricorda la luce naturale e spesso soffusa ammirata nelle fotografie di Ghirri, o la «luce scoppiata» della novella «Esplorazioni sulla via Emilia». Questa luce, che Celati confronta nelle fotografie di Gajani alla «pittura di paesaggio di altri tempi o (se volete) della poesia giapponese» (350), è contrapposta alla luce squillante del realismo documentario di certa fotografia americana contemporanea, che Ghirri in un saggio del 1984 su William Eggleston contrasta con il Lento ritorno di Sorger, il protagonista dell'omonimo romanzo di Handke (Ghirri 1997: 52). Come per la narrativa, la fotografia qui rappresenta non un «paesaggio idealizzato», ma «una possibilità del pensiero di individuare qualcosa al di là o prima dello sfacelo» (Celati 2017c: 351) - frasi consone alla poetica celatiana del tempo, improntata al pensiero archeologico e benjaminiano. Come nei commenti su Ghirri, e in tono ugualmente lirico, Celati sottolinea l'aspetto poetico della fotografia di Gajani, nella sua capacità di rappresentare «le brume dei vapori esalati dalle piante» (352), di «pensare con rispetto al mistero che c'è al di là di ogni soglia, al carattere fiabesco d'ogni architettura non databile, alla calma delle cose, al sentimento delle ore del giorno» (352), e di offrire non solo uno «sfondo in [sic!] cui proiettare il pensiero» ma anche «una dimora a cui apparteniamo come le piante» (352). Se 
Gajani è celebrato «tra tutti i fotografi che conosco» come «quello che più mi richiama ad esigenze del genere» (352), dopo poco questo ruolo viene affidato a Ghirri, e Gajani non viene più menzionato negli scritti celatiani, tranne un breve appunto del 1985 nella busta 12/155 (14 aprile).

Nonostante la consonanza di vedute tra gli scritti celatiani su e per Gajani e quelli su e per Ghirri, è interessante notare come il dialogo interartistico tra letteratura e fotografia non dia necessariamente luogo ad un dialogo concreto delle due arti sulla pagina. Infatti, molti dei progetti celatiani di fotolibro, prima con Gajani poi con Ghirri, rimangono incompiuti. Con fotolibro qui intendo quella che Vangi definisce «modalità iconotestuale», in cui «l'immagine fotografica non è solo tematizzata o simulata nel testo letterario, ma vi compare fisicamente sotto forma di riproduzione» (Vangi 2005: 40). Il sopracitato progetto «Disegni di paesaggio. Viaggio fotografico nelle pianure dell'ultimo Po» non viene completato e segna la fine dei rapporti tra Celati e Gajani ed il passaggio alla collaborazione con Ghirri, sempre sul paesaggio padano. Similmente, il fotolibro progettato con Ghirri in quegli anni alla fine non va in porto, ma dà luogo a due progetti paralleli, pubblicati entrambi nel 1989, Il profilo delle nuvole di Ghirri e Verso la foce di Celati - quasi a testimoniare l'impossibilità di una totale coerenza di visione, o, come spesso accade, di un fotolibro che sia all'altezza di ognuna delle sue componenti, come ci ricorda Jefferson Hunter in Image and Word. Celati rivela una veduta simile in una bozza di intervista con Daniel Gunn per The Cambridge Review, conservata presso il Fondo Gabriele e Gianni Celati, dove parla di un progetto incompiuto di descrizione del paesaggio scozzese, che doveva scaturire in un altro fotolibro ${ }^{5}$, come si evince anche dal riferimento ad un "diario scozzese» in un appunto inedito del Fondo Celati (FC 11/155). Il dattiloscritto non datato (n. 7 del Fondo) fa riferimento alla pubblicazione «later this year» di un «travel log about the Po river», "subsequently extended» (2), ed è quindi databile al 1989, anno della pubblicazione in volume di Verso la foce. Celati parla dal lavoro con Ghirri sul paesaggio padano, un paesaggio ritenuto «ugly» (1), orribile, e pertanto spesso ignorato, ma che i due intendono recuperare e guardare con occhi nuovi: «neither in terms of objective documentation, nor in terms of subjective contemplation» (1). Lo scopo ultimo è di scovare il "destino" di questo paesaggio ${ }^{6}$, cioè vederne la trasformazione in atto. Questo sguardo sui luoghi qualsiasi è presentato come eccezione alla maniera occidentale di guardare lo spazio, ma non è iscritto in una tradizione visiva - fotografica o artistica - bensì letteraria, che per Celati include pochi scrittori: Conrad, Hardy, forse Flaubert. E interessante inoltre notare che, pur citando Ghirri in apertura, Celati non

5 Fondo Gabriele Celati, Interview with Gianni Celati by Daniel Gunn, forthcoming on The Cambridge Review, dattiloscritto di 5 pagine numerate.

6 Celati spiega: «By "destiny" I mean the possibility of seeing what is currently happening, and what a landscape is doomed to be» (Fondo Gabriele e Gianni Celati, dattiloscritto n. $7: 1$ ). 
parla di suoi progetti specifici, ma della collaborazione per Viaggio in Italia, iscrivendo il lavoro dei venti fotografi nella linea ghirriana, e all'interno di una più ampia tradizione di arti visive che include De Sica, Zavattini, Paul Strand, Walker Evans, ed anche i primi film di Antonioni e di Visconti per lo sguardo su un paesaggio «non interessante», fino ad arrivare a Wenders e a Calvino. In linea con Hunter, Celati nega un'interazione pratica tra la fotografia e il testo scritto ma parla di un atteggiamento comune che identifica nella «descrizione», presa non in senso scientifico, ma come ausilio all'immaginazione ${ }^{7}$.

Nell'avvicinare, seppure per contrasto, il paesaggio scozzese, come il meno americanizzato del mondo occidentale, un paesaggio che conserva ancora tracce del passato e una sembianza di futuro, a quello Padano in via di sparizione, Celati parla dell' «estuario di un'epoca» facendo riferimento a Verso la foce e insiste sulla «cura» che occorre dedicare ai luoghi. Per l'autore sia che si scriva o si faccia fotografia, due operazioni sono possibili: sfruttare oppure prendersi cura dell'ambiente. Emerge qui un Celati ambientalista che associa le due arti come mezzi paralleli per tentare di salvaguardare l'ambiente. Nonostante la comunanza di intenti e la consonanza di pratiche estetiche, il dialogo sulla pagina tra le due arti rimane alla fine un ideale, e il fotolibro una possibilità remota: «if possible, one could make a book, incorporating texts and photographs, which would surely be interesting, because it is something that is rarely if ever done» (5). Alla domanda su quali vorrebbe fossero i risultati del suo progetto sul paesaggio scozzese, Celati risponde descrivendo un approccio simile a quello per Strada provinciale delle anime:

A group of people visiting different areas of Scotland, or already living there, taking notes, observing and trying to describe what is going on. Trying to create an image of this land which should not be consolatory or folkloristic or journalistic or aesthetic. Trying to find a way of looking at things with different eyes, and different emotions, rediscovering the earth, the soil, and the ways of inhabiting it. (5)

Come per i lavori con Gajani e con Ghirri, similmente per il progetto inedito sul paesaggio scozzese il fotolibro sembra un ideale a cui tendere, più che una prospettiva reale di dialogo tra scrittura e fotografia sulla pagina, ma ciononostante testimonia la coerenza della riflessione celatiana sulla fotografia come pratica del pensare per immagini, e come mezzo privilegiato per descrivere luoghi in via di sparizione, in parallelo alla scrittura.

Partendo dalla frequentazione con il lavoro di Gajani e di Ghirri, Celati sfrutta la fotografia per rimodellare la sua scrittura negli anni Ottanta e con essa il suo approccio estetico alla visione dell'esterno quale "pratico pensare per immagini», come superamento di una «misura scalare» del visibile, verso una lingua che

7 Nelle parole di Celati: “"Description” is the key term, for normally we think of description as being something scientific. Photographs and writing should, I believe, be used in such a way as to help people to imagine what is existing in the space around them» (Fondo Gabriele e Gianni Celati, dattiloscritto n. 7: 3). 
lasci le cose nella loro distanza, senza nominarle, e inviti all'immaginazione. Ciò emerge in vario modo nella trilogia degli anni Ottanta e in particolare nel palinsesto che è Verso la foce, a detta dello stesso autore il testo che «mi ha più cambiato e che mi ha più impegnato» (Celati in Belpoliti e Cortellessa 2008: 36), e che ha lasciato un forte impatto sia sulla letteratura che sulla fotografia contemporanee. Come è noto, Verso la foce viene pubblicato in prima versione come introduzione al volume fotografico Viaggio in Italia e fin dall'origine si presenta come testo ibrido - reportage/documentario, diario di viaggio e racconto d'osservazione a un tempo - e come «un punto limite» (36) per l'autore nel trovare una Stimmung, un reincanto per zone di desolazione post-industriale, e nell'allontanarsi dall'idea di letteratura comunemente accettata. Un'analisi dei manoscritti del Fondo Celati - in particolare degli appunti sulle pagine di aprile nell'agenda preservata nel contenitore 12/145, scritti in Sicilia nell'agosto 1984, e sulle pagine di giugno della busta 10/86 - evidenzia un lungo processo di ridefinizione generica del testo e quindi dell'autore stesso - processo che, come ho dimostrato in un recente saggio (Spunta 2019a), va da un'opposizione dicotomica di generi diversi, sottolineata dall'asserzione di Celati: «Questa non è letteratura [...] è un reportage sulla visione che abbiamo dei posti» (Celati in Belpoliti e Cortellessa 2008: 32) - verso una coesistenza dialettica degli stessi, come emerge nel primo paragrafo della Notizia introduttiva al testo del 1989, che lo definisce a un tempo come «diari di viaggio» e «racconti d'osservazione» (1989a: 9). Tale processo di riscrittura va di pari passo alla riflessione celatiana sulla fotografia, sull'immagine e sulla visione, e al lavoro con Gajani, Ghirri e altri fotografi, e dimostra come il discorso celatiano sulla fotografia informi la sua scrittura e sia un mezzo per riflettere sulla stessa.

L'interstualità con la fotografia in Verso la foce emerge sia a livello macrotestuale nel definire Verso la foce come coesistenza di generi diversi, sia a livello microtestuale nei continui riferimenti alla distanza, alla vaghezza, alla luce, e alla difficoltà di rappresentare il paesaggio verbalmente o visivamente. Accompagnato a turno dal fotografo Luciano Capelli («Esplorazioni sugli argini») e dal fotografo/documentarista svizzero Reinhard Dellit («Verso la foce»), il narratore in Verso la foce sperimenta ripetutamente l'impossibilità di mappare un territorio vago, contingente, a un tempo ovvio e suggestivo, narrabile solo nel momento in cui ci si scorda il «bisogno di fotografare» e il desiderio di «fotografare tutto» (FC 12/167, 21 maggio), ma immaginabile solo grazie alla misura scalare data dalla fotografia: «Luciano mi spiega che solo immaginando un pezzo di carta lucida su cui tutte le apparenze disperse si collegheranno grazie a piani prospettici, è possibile per lui vedere qualcosa là fuori; attraverso l'obiettivo osserva a occhio nudo le nuvole che si formano o disperdono» (FC 12/167, 26 maggio). Se lo sguardo documentario, come quello fotografico, sembra essere privilegiato nei confronti della parola, in quanto capace letteralmente di "pensare per immagini», non costretto dalla lingua a nominare le cose - come emerge nel sopracitato brano della prima versione di Verso la foce, espunto nelle versioni successive - sia il fotografo che lo scrittore 
risultano ben presto sconfitti da questo territorio; a conclusione del primo nucleo narrativo nella versione del 1984, e di «Esplorazioni sugli argini» nel testo del 1989, Luciano improvvisamente sbotta: «Non capisco niente di cosa c'è intorno, di cosa fotografo, di cosa fa la gente. [...] Torno a casa» (Celati 1984: 22).

Nell'intervista con Marco Sironi, Celati ammette che «L'intento primo di Verso la foce, più che un discorso di tipo sociale o geografico» era «il fatto primario che gli aspetti di un luogo li cogliamo come apparizioni» (Celati 2004d: 221); «il mio problema [...] era appunto questo: smontare l'apparato discorsivo che avvolge i luoghi, e ricondurli al fenomeno del "vedere", dell'apparizione». E continua: "Ghirri ha fatto la stessa cosa con il suo lavoro fotografico veramente unico, che finalmente ha tolto di mezzo i precetti del documentarismo sociale: precetti e discorsi che bloccano la visione su un tipo di oggettività astratta» (223), dando luogo a «un'infinità di mondi immaginari» (Ghirri 2006: 36). Qualcosa di simile accade nella scrittura celatiana di Verso la foce nell'oscillare tra l'annotazione diretta (con descrizioni più estese) al raccontare a distanza, oscillazione più forte nella prima versione del testo, l'introduzione a Viaggio in Italia - nel montaggio di brani che poi confluiscono in diverse sezioni di Verso la foce e in tre racconti di Narratori delle pianure - che viene poi armonizzata nella coesistenza di generi diversi nella versione finale.

Nel saggio "La fotografia dei luoghi come fotografia», Roberta Valtorta sostiene che l'osservazione del paesaggio fatta da Ghirri e colleghi per il progetto Viaggio in Italia "prende [...] il significato soprattutto di una verifica dell'operazione fotografica» (2005: 246) e dà una nuova direzione alla fotografia italiana. Similmente, per Celati la collaborazione con Gajani e con Ghirri e la riflessione sulla fotografia, sulla visione e sulle immagini, in vari testi critici e narrativi tra cui Verso la foce, diventa un modo per riflettere non solo sul paesaggio, ma anche sulla propria scrittura, nel tentativo di passare dall' «idea» alla «pratica» della letteratura (Celati in Rasy 1989: 25) e di «farsi più prossimi al mondo esterno e alle cose che abbiamo davanti» (Celati in Pomilio 2008: 193). Ciò grazie alla descrizione, che Celati nell'intervista con Daniel Gunn pone come l'atteggiamento comune alle varie arti, quale ausilio all'immaginazione. Se Gajani, a detta di Celati, lo richiama ad un paesaggio che sia dimora per l'uomo, similmente, sempre secondo Celati, "Ghirri ci ha aiutato come pochi a capire che il confronto tra il nostro pensiero e il mondo esterno non consiste in una registrazione di fatti, [ma] in un lavoro immaginativo [che è] implicato nelle abitudini di tutti» (2004c: 205) - un approccio che possiamo leggere in parallelo al recupero della "finzione" nella ridefinizione generica di Verso la foce che emerge dai manoscritti. Oltre a suggerire il superamento di una misura scalare, e l'anti-letterario in letteratura, evidenziandone la natura porosa, come suggerisce Vangi (2009: 91), il riferimento alla fotografia come "pratico pensare per immagini» serve inoltre a Celati a scardinare il primato della visione o il paradigma oculo-centrico occidentale, aprendo il senso/significato a una pluralità di sensi - e senso è qui inteso con Adrienne Janus (2011: 183) nell'accezione tripartita quale senso/significato, direzione di marcia (che in 
Celati, come in Ghirri, si apre in mappa), e percezione sensoriale, a partire dal multidirezionale udito, o ascolto, quale attività «non direzionale, univoca», che "produce continuamente [...] nuovi [significati], senza mai fissare il senso» (Barthes e Havas 1977: 982). Oltre alla fotografia, infatti, l'ascolto è una metafora chiave nella pratica e nella poetica celatiana, con cui non a caso si chiude Verso la foce, mandando un ultimo richiamo - a un tempo sonoro, verbale e visivo verso le cose, prima che svaniscano.

\section{Bibliografia}

AA. VV., 1989, Il racconto del nostro presente, Firenze, Alinari.

Adams A., 1986, Ansel Adams, Milano, Idea Books.

Arborio Mella F., 1976, Sulla strada della fotografia, Milano, Feltrinelli.

Barthes R., [1975] 1980, Barthes di Roland Barthes, traduzione di G. Celati, Torino, Einaudi.

Barthes R., [1980] 1980, La camera chiara: nota sulla fotografia, Torino, Einaudi.

Barthes R. e Havas R., 1977, «Ascolto», in Enciclopedia Einaudi. Vol.1, Torino, Einaudi, p. 982-991.

Bartezzaghi S., 2012, "Celati fra le nuvole», Nuova Prosa, 59, Il comico come strategia in Gianni Celati \& Co., p. 37-47.

Belpoliti M. e Cortellessa A., 2008, «Letteratura come accumulo di roba sparsa», Riga, 28, Gianni Celati, a cura di M. Belpoliti e M. Sironi, p. 24-44.

Berger J., 1978, «Uses of Photography», in Id., 2001, Selected Essays, a cura di G. Dyer, London, Vintage, p. 286-293.

Calvino I., 1979, Se una notte d'inverno un viaggiatore, Torino, Einaudi.

Calvino I., 1983, Palomar, Torino, Einaudi.

Calvino I., 1988, Lezioni americane, Milano, Garzanti.

Celati G., 1979, «Oggetti soffici», Iterarte, numero monografico su Arte affettuosa, 17, a cura di C. Gajani, p. 10-15.

Celati G., 1984, "Verso la foce (reportage per un amico fotografo)», in Ghirri, Leone e Velati, 1984, p. 20-35.

Celati G., 1985, Narratori delle pianure, Milano, Feltrinelli.

Celati G., 1987a, Quattro novelle sulle apparenze, Milano, Feltrinelli.

Celati G., 1987b, "Verso la foce (estratti da un diario di viaggio)», in Narratori dell'invisibile. Simposio in memoria di Italo Calvino, a cura di B. Cottafavi e M. Magni, Modena, Mucchi, p. 65-79.

Celati G., 1989a, Verso la foce, Milano, Feltrinelli.

Celati G., 1989b, "Commenti su un teatro naturale delle immagini», in Ghirri 1989.

Celati G., 1991, Strada provinciale delle anime, DVD.

Celati G., 2001, Cinema naturale, Milano, Feltrinelli.

Celati G., 2003, «Collezione di spazi», il verri, 21, p. 57-92. 
Celati G., 2004a, «Viaggio in Italia con venti fotografi, vent'anni dopo», in Valtorta 2004, p. 74-86.

Celati G., [1992] 2004b, «Soglia per Luigi Ghirri: come pensare per immagini», in Sironi 2004, p. 190-194.

Celati G., 2004c, «Luigi Ghirri, leggere e pensare per immagini», in Sironi 2004, p. 205-209.

Celati G., 2004d. «Qualche idea sui luoghi e il lavoro con Luigi Ghirri. Intervista con Marco Sironi», in Sironi 2004, p. 221-229.

Celati G., 2017a, Animazioni e incantamenti, con C. Gajani, a cura di N. Palmieri, Roma, L'Orma.

Celati G., [1983] 2017b, «I viandanti osservatori», in Celati 2017a, p. 345-348.

Celati G., [1985] 2017c, «Brani di natura nella valle del Po», in Celati 2017a, p. 349-352.

Celati G., 2017d, «Pedinando Zavattini», L'Indice dei libri del mese, 5, p. 24.

Costantini P., Fuso S. e Mescola S. (a cura di), 1987, Nuovo paesaggio americano/ Dialectical Landscapes, Milano, Electa.

Cox C. (a cura di), 1981, Dorothea Lange, New York, Aperture Masters of Photography, n. 5, Aperture.

De Seta C. (a cura di), 1981, Napoli '81. Sette fotografi per una nuova immagine, Milano, Electa.

Dorfles G., 1978, «Come pensare per immagini. Una disputa su percezione e realtà», Il Corriere della Sera, 17 agosto, p. 3.

Fameli P., 2017, «Celati e Gajani in dialogo», in Celati 2017a, p. 441-451.

I figli del deserto di Fusignano (a cura di), 1986, Traversate del deserto, Ravenna, Essegi.

Gajani C., 1984, «Disegni di paesaggio. Viaggio fotografico nelle pianure dell'ultimo Po», a cura di R. Renzi, FG, dattiloscritto, 15 Giugno, p. 1-3.

Gajani C., 1985, Carlo Gajani. Brani di natura nella valle del Po, Bologna, Grafis.

Gajani C., 2010, Carlo Gajani. 1929-2009, a cura di C. Casarin, Bologna, Edizioni Alma.

Ghirri, L., 1989, Il profilo delle nuvole, Milano, Feltrinelli.

Ghirri, L., 1997, Niente di antico sotto il sole. Scritti e immagini per un'autobiografia, a cura di P. Costantini e G. Chiaramonte, Torino, Società Editrice Internazionale.

Ghirri, L., [1982] 2006, Still life, in Luigi Ghirri. Del guardare, a cura di I. Ghirri e P. Ghirri, Milano, Baldini Castoldi Dalai.

Ghirri, L., 2010, Lezioni di fotografia, a cura di G. Bizzarri e P. Barbaro, Macerata, Quodlibet.

Ghirri L., Leone G. e Velati E. (a cura di), 1984, Viaggio in Italia, Alessandria, Il Quadrante.

Guidi G., «Una fotografia della qualsiesità», in Valtorta 2004, p. 178-180.

Handke P., [1977] 1981, Il peso del mondo, Milano, Guanda. 
Handke P., [1979] 1986, Lento ritorno a casa, Milano, Garzanti.

Hunter J., 1987, Image and Word: The Interaction of Twentieth Century Photographs and Texts, Cambridge, Mass., Harvard University Press.

Janus A., 2011, «Listening: Jean-Luc Nancy and the "Anti-Ocular" Turn in Continental Philosophy and Critical Theory», Comparative Literature, 63, 2 (Spring), p. 182-202.

Jeffrey I., 1991, Photography, Londra, Thames and Hudson.

Magni M., 2004, Viaggio in Italia. I fotografi vent'anni dopo, DVD.

Marra C. (a cura di), 2001, Le idee della fotografia. La riflessione teorica dagli anni Sessanta a oggi, Milano, Mondadori.

Messori G., 1992, «Fin dove può arrivare l'infinito?», in Luigi Ghirri. Vista con camera. 200 fotografie in Emilia Romagna, a cura di P. Ghirri e E. Taramelli, Milano, Motta, pp. 193-194.

Messori G., 2004, «Il mio incontro con Viaggio in Italia», in Valtorta 2004, p. 102105.

Mulas U., 1973, La fotografia, a cura di P. Fossati, Torino, Einaudi.

Orlandi P., 2014, Visioni di Città. La fotografia tra indagine e progetto, Bologna, Bononia University Press.

Palmieri N., 2017, «Il gesto, gli oggetti, le immagini. Gianni Celati nell'animazione del mondo», in Celati 2017a, p. 421-438.

Pomilio T., 2008, «Ovvietà, ultima rivelazione», Riga, 28, Gianni Celati, a cura di M. Belpoliti e M. Sironi, p. 193-194.

Rasy E., 1989, "Gianni Celati, "Verso la foce”», Nuovi Argomenti, 32 (Ott-Dic), p. 123-125.

Sironi M., 2004, Geografie del narrare, Insistenze sui luoghi di Gianni Celati e Luigi Ghirri, Reggio Emilia, Diabasis.

Spunta M., 2017a, «Narrating the experience of place: Luigi Ghirri and literature», in Luigi Ghirri and the Photography of Place. Interdisciplinary Perspectives, a cura di M. Spunta e J. Benci, Oxford, Peter Lang, p. 199-244.

Spunta M., 2019a, «Verso la foce: The Experience of Place between Writing and Photography», in Towards the River's Mouth (Verso la foce) by Gianni Celati. A critical Edition, a cura di P. Barron, Lanhan, Maryland, Lexington Books, p. 77-88.

Spunta M., 2019b, «Gianni Celati and the allure of the voice: from "Voci da terra" to "Notizie ai naviganti"», in Gianni Celati. Traduzione, tradizione e riscrittura, a cura di M. Ronchi Stefanati, Canterano, Aracne, p. 49-68.

Strand P. e Zavattini C., 1955, Un paese, Torino, Einaudi.

Taramelli E., 2005, Mondi infiniti di Luigi Ghirri, Modena, Diabasis.

Thompson J., 2016, Why Photography Matters, Cambridge, Mass, The MIT Press. Valtorta R., (a cura di), 2004, Racconti dal paesaggio. 1984-2004. A vent'anni da Viaggio in Italia, Milano, Lupetti, p. 102-105. 
Valtorta R., [1997] 2005, «La fotografia dei luoghi come fotografia», in Volti della fotografia, a cura di R. Valtorta, Milano, Skira, p. 109-135.

Valtorta R., (a cura di), 2013, Luogo e identità nella fotografia contemporanea, Torino, Einaudi.

Vangi M., 2005, Letteratura e fotografia, Pasian di Prato, Udine, Campanotto Editore.

Zannier I. e Costantini P. (a cura di), 1989, 150 anni della fotografia in Italia. Un itinerario, Roma, Regione Lazio. 